\title{
When health means suffering: mammograms, pain and compassionate care
}

\author{
N. MORRIS, phD, ReSEARCh fellow, Department of Science and Technology Studies, University College London, \\ London, UK
}

\section{MORRIS N. (2014) European Journal of Cancer Care \\ When health means suffering: mammograms, pain and compassionate care}

\begin{abstract}
The X-ray mammogram remains the cornerstone of most public health programmes aimed at the early diagnosis of breast cancer. Its virtues of safety, reliability and cheapness maintain its established position, and Western social and cultural traditions of ambivalence to pain push any questions concerning the painfulness of the procedure into the background. As part of a larger UK/USA-based empirical study, we undertook a qualitative analysis of women's accounts of pain experienced in mammograms and their reaction to it, comparing their accounts with professional views and advice to patients as reflected in interviews, patient leaflets and practice guidelines. We found considerable variability of experience and reaction to pain among patients, and indications of similar variability in professionals' views and practice, contrasting with a uniformly reassuring message in formal institutional advice. We suggest that in practice professional workarounds and patients' felt obligation to tolerate pain bridge this gap, but that action to tackle the problems of dropout and the emotional and operational costs of the current system is nonetheless needed. The need is for concerned groups to combine to establish a serious and sustained programme of amelioration and innovative technological development to assure more compassionate patient care and operational efficiency.
\end{abstract}

Keywords: mammograms, pain, patient experience, breast cancer diagnosis, screening programmes, public policy.

\section{INTRODUCTION}

Cancer is recognised as a major public health risk in most advanced societies, and national health-care policies actively promote early diagnosis and treatment. For breast cancer, the mammogram is well established as the principal instrument for early diagnosis and is widely used in mass screening programmes as well as in clinical diagnosis. Mammogram use is not without controversy, with

Correspondence address: Norma Morris, Department of Science and Technology Studies, University College London, Gower Street, London WC1E 6BT, UK (e-mail: norma.morris@ucl.ac.uk).

Funding: UK Economic and Social Research Council (ESRC) Grants RES000-22-0093 and RES-000-23-1160.

Accepted 4 November 2014

DOI: $10.1111 /$ ecc. 12272

European Journal of Cancer Care, 2014 concerns centring principally on diagnostic accuracy, risks from radiation and 'overdiagnosis', leading to unnecessary treatment (Jørgensen et al. 2011). Amidst these concerns and controversies, there has been little room for critical consideration of the physical demands of the procedure itself and what that means for patients. Yet there is a background rumble of discussion in the scholarly literature and less formal sources, which suggests that pain in mammography is not a negligible issue for patients and not without costs.

The present paper focuses on this relatively neglected topic, using new and existing data to probe the strategies, alliances, rationalisations and $a d$ hoc negotiations that are used to manage pain (or contest its existence) at the individual, collective and institutional level, with a particular focus on patient experience and views. Pain in mammograms emerges as an exemplar of an area of intersection

(C) 2014 The Author. European Journal of Cancer Care published by John Wiley \& Sons Ltd.

This is an open access article under the terms of the Creative Commons Attribution License, which permits use, distribution and reproduction in any medium, provided the original work is properly cited. 
between issues at both the individual and institutional level. At the individual level, issues of 'moral obligation' (both as responsible citizens, and a female duty) meet issues of personal and patient freedom of choice in health care. At the institutional level, organisational practices of safety (in assuring clinical diagnosis or public health) intersect and may conflict with policies of compassionate and personalised care. The empirical evidence suggests that despite our supposedly pain-averse society, and the demonstrable tensions pain induces, pain is the loser in any contest between compassionate and strictly compliance-oriented approaches. It is concluded that the ambivalence of both patients and professionals towards pain results in weakening of any collective will to stimulate radical change; but their muted protests nevertheless deserve more enlightened consideration by ethicists, health-care authorities and research funders through programmes of mitigation and future development.

\section{PRIOR LITERATURE}

\section{Social and cultural dimensions of pain}

We frame our understanding of the approaches of different actors to questions of pain in diagnosis and treatment by reference to three principal inter-relating influences: conceptual issues over pain in Western medical practice, socio-political pressures relating to good citizenship and relations of authority, as discussed below.

Pain has long been a contested issue in Western medicine. In a recent report deploring inadequate standards of treatment for chronic pain in the USA, the Institute of Medicine (IoM) endorsed the long-standing critique of the inability of Western medical culture, rooted in a Cartesian mind-body dualism, to move beyond narrow physiological definitions of pain. IoM refers to increasing acknowledgement within the profession that pain cannot be reduced to biology alone, but finds a continuing failure to translate this into practice (IoM 2011). Similar arguments and concerns around broadening understanding of the interweaving of mind and body in the phenomenon of pain are heard in other nation states (Scheper-Hughes \& Lock 1987; Baszanger 1998; Resnik \& Rehm 2001; Crowley-Matoka et al. 2009). There is thus a longstanding tension between popular and different professionals' understandings of what may count as pain.

Tensions, and cultural differences, likewise exist over the place of pain in society. The culture of contemporary Western society is generally strongly averse to pain, but yet allows certain 'culturally authorised' exemptions, including tolerance of pain in restoring health or for bodily improvement (Shilling \& Mellor 2010). The cultural exemption for pain in the context of expected health benefits slots into place within a further authoritative contemporary cultural discourse on individual citizens' duty to take responsibility for their own health, as part of 'biological citizenship' (Rose \& Novas 2007). This discourse, building on the Foucauldian concepts of governmentality, technologies of self, biopower and biopolitics, is shaping power relations between the state and its citizens (Armstrong 1995; Petersen \& Bunton 1997; Rose 2001) and is important for the operation of public health programmes promoting early diagnosis or lifestyle interventions (Howson 1999; Armstrong et al. 2007; Roy 2008; Department of Health 2010; Kampf 2010). Citizens' adaptation to this regime is not without stresses and defaulters, as the case of pain in mammography will illustrate.

For the patient reporting for a breast screen or diagnosis of a suspicious lump, the authority of government that supports the discourse of responsible citizenship, by urging citizens to be risk aware and take responsibility for their health (Department of Health 2009, 2010), coalesces with the medical authority supporting mammography technology. This is further reinforced by unconditional endorsement of these dicta by cancer charities and patient groups, thus making a powerful alliance covering virtually all sources of authoritative information patients might seek and leaving scant room for expression of patient concerns about pain.

\section{Academic literature}

Although medical advice to patients remains that having a mammogram may be uncomfortable but is rarely painful, there is no lack of medical and public health literature on pain in mammograms. This literature shows both sharp differences of opinion between professionals and widely varying accounts of patients' views. Studies that attempt quantitative measurement of how much, if any, pain is felt by women undergoing mammography have failed to produce consistent findings, with reported incidence of pain (variously defined) varying between $1 \%$ and 93\% (Davey 2007), with a substantial part of that variation being attributable to choice of survey instrument (Kornguth et al. 1996; Kashikar-Zuck et al. 1997). While some early studies concluded that pain is not a problem, later reviews indicate that many others express concern (Andrews 2001; Davey 2007; Miller et al. 2008). Another focus of study is increased risks of noncompliance if women fear the mammogram and expect it to be painful (Marshall 1994; Elwood et al. 1998; Doyle \& Stanton 2002; Poulos \& Llewellyn 2005; Miller et al. 
2008). A further strand of investigation seeks to identify, using survey methods, demographic or operational factors associated with pain and to propose practical ways for mitigation within existing frameworks (Davey 2007; Miller et al. 2008), although few, if any, of these have found their way into practice.

\section{Grey literature}

In contrast to these mixed views, the 'grey' literature of pamphlets and web pages offering authoritative medical advice to patients from health providers, cancer charities and patient associations is virtually unanimous in dismissing any pain as trivial. For example, the leaflet offered by the UK National Health Service (NHS 2011) - used for both screening and clinical assessment patients - states in answer to the question 'Does having a mammogram hurt?':

Most women find having a mammogram uncomfortable. Some women find it painful, but only for a few seconds. (p. 5)

By making a distinction between painful and 'uncomfortable', the leaflet implies no pain for the 'normal' woman. Additionally, emphasis is put on the transience of pain, without discussion of its quality, or the need for more than one exposure. A similar assumption that the normal woman will experience at most 'discomfort' appears in patient information in Australia and Canada (BreastScreen Australia 2014; Screening for life.CA 2014). The influential American Cancer Society (ACS) similarly advises:

Although the compression can [be] uncomfortable and even painful for some women, it only lasts for a few seconds and is needed to produce a good picture.

$(\text { ACS 2014) })^{1}$

The authoritative messages coming both from service providers and major cancer charities such as ACS and Cancer Research UK (which co-authors the UK NHS leaflet) as to the triviality or non-existence of pain are further reinforced by smaller charities and patient associations, which here as elsewhere generally align themselves with the medical establishment (Jørgensen \& Gotzsche 2004; Brown et al. 2011; ACS 2014).

${ }^{1}$ http://www.cancer.org/healthy/findcancerearly/examandtest descriptions/mammogramsandotherbreastimagingprocedures/ mammograms-and-other-breast-imaging-procedures-having-a -mammogram

\section{Social science studies}

There is in addition one substantial book-length critique from a social science perspective, arguing that mammography is an inherently unsuitable technology in need of replacement (Cartwright 1995), and a growing number of qualitative studies covering patient or consumer perspectives. These will be considered alongside new empirical data from a series of qualitative interviews to be considered in the next section.

\section{METHODS}

A qualitative methodology was chosen to throw light on women's reasoning about mammograms and the demands of the procedure. Collaborative studies had already been set up with two medical physics teams running clinical tests of novel, optics-based technologies with potential for diagnosis of breast cancer to study women's experience of health technologies and of research. A team of three social scientists interviewed 85 participating women 165 in the UK, 15 in the USA). Most of the women were attending a breast clinic in a university hospital complex (and recruited by collaborating clinical staff on criteria pertaining to the medical physics test protocol), and about one fifth were healthy volunteers (recruited through the medical physicists' personal or institutional networks). Interviews, which took place in university accommodation after the women underwent an experimental breast scan, were semi-structured, with space for patient narrative. Typically, an interview would include prompts to the patient to talk about how she had felt about taking part in the experimental scan (before, during and after), how she would describe the experience, prior experience of breast examinations or research, her views on health technology and on research participation in general, and any thoughts she had about the usefulness and appropriate priority for innovations of this kind. Mammograms were not listed as a topic in the interview schedule but emerged as important from patient narratives. In particular, two of the topics routinely covered tended to trigger sometimes copious commentary: (1) participants' evaluation of their experience of the experimental scan; and (2) the enquiry about prior experience of breast examinations. To assist in the analysis of this unscheduled data, three additional interviews were arranged with two health professionals at a diagnostic centre and a patient advocate.

Interviews were digitally recorded, subject to participant consent, and transcribed verbatim. Data analysis followed standard qualitative methods, with iterative reading of transcripts, by all social sciences team members to assist in development of a coding frame, and use of 
Atlas-ti software (Atlas.ti Scientific Software Development $\mathrm{GmbH}$, Berlin, Germany) to assist data management and analysis. In approaching the analysis, we were mindful that interviewees' accounts actively construct rather than simply describe their experience and their motivations (Mills 1940; Potter 1996; Gill 2000).

Ethical approval was obtained from the local Research Ethics Committees in the UK and in the USA. The code used below for distinguishing participants (while preserving anonymity) is a number preceded by UK/USA to differentiate the UK and US cohorts, and PV or HV to indicate whether patient-volunteer or healthy volunteer.

\section{FINDINGS}

Participating patients' views on pain in mammograms although not homogenous share common themes (whether screening or clinical diagnostic mammograms are at issue). The published and unpublished data cited below provide material for a qualitative understanding of participants' concerns and personal stresses as expressed in patients' narratives, and the kinds of resources they and professionals need to mobilise to deal with the conflicts in practice.

\section{Vocabularies of pain}

The language used by patients to describe the experience of the mammogram was often highly emotive and contrasted with the measured prose of the patient information, going well beyond expected differences between published documents and oral communication in an interview. Terms used to describe the sensation in the breasts included 'squashed', 'squished', 'squeezed', 'mashing' and 'smashing'; while the process was 'terrible', 'excruciating', 'a shock', 'agony', 'a vice' and 'like torture'. Other studies, in the USA, Australia and Sweden record similar language, for example, 'cramming, squashing, squeezing' (Engelman et al. 2006); being 'squashed to paper thin' (Poulos \& Llewellyn 2005); and 'tormenting, torturing' (Johansson \& Berterõ 2003). A more moderate tone was used by a veteran cancer survivor taking part in my study:

Patient: You've had a mammogram?

Interviewer: No, I've never had one.

Patient: Okay, it, it hurts. They clamp it and they make it so tight. I can't have one on this side now, with the implant, . . . but it can really hurt. They push in from here, and they push in from there. ... It's, it's very hard. (UKPV40)

Whereas these terms emphasise the physical hurt of the procedure, the women's language also includes terms like 'yank', 'invasive' and 'humiliating', signalling the importance of the 'non-biological' aspects of pain, still not always sufficiently recognised in everyday practice. This is a particular issue in the context of the mammogram, dealing as it does with a tissue that is culturally, as well as physically highly sensitive, and where touching or even exposure can cause embarrassment or humiliation. 'Yanking' of the breast implies not only pain, but also depersonalisation of the patient to merely material for processing:

It's like they're handling a lump of meat. Sort of throwing it on a slab and doing something to it. (Poulos \& Llewellyn 2005; see also Pfeffer 2004).

it's a bit, you know, sort of, putting your boob, plonk it on a plate, you know [laughter] as if it's a piece of, piece of meat sort of thing. (UKHV8)

Clinical diagnostic mammograms similarly evoke a range of painful emotions:

With the mammogram, you're thinking, oh, it's going to hurt, they're going to squash it, it's embarrassing, someone's touching my breast, moving it around. (UKPV5)

\section{Variability and ambiguity}

There is no consensus on how widespread such sensations are, but the literature supports the conclusion of wide variability among women in pain sensitivity, coping strategies and sensitivity to context (Kashikar-Zuck et al. 1997; Brett et al. 2005), as does available interview data. Some women, while expressing sympathy for others, report no personal problems:

I've never experienced, you know, pain and problems that other people complain about. (IIPV28) or

I don't find them unpleasant. It's just one of those things. (UKHV3)

- although in the latter statement, the second part seems rather like a qualification of the first. There is also use of an apparently deliberately understated language:

Uncomfortable. Not a pleasant experience. (UKHV1)

Not the most comfortable thing in the world. (USHV84)

These women may want to emphasise their strength, as reported for women with chronic pain (Werner et al. 2004), but in some instances participants using almost the same language do connect it to pain, for example, 
... not a very pleasant experience, I must say ... it's quite a shock ... the squashing and the squeezing ... and the pain. (UKPV29)

always painful, um, uncomfortable. (USPV87)

These examples illustrate some of the difficulties besetting surveys enquiring about levels of pain/discomfort and also begin to suggest how women strategically position themselves to exert control over their pain experience.

\section{Strategic positioning}

The interviews showed some of the devices women used to help them feel, and show themselves as in control of pain. Some used humour, laughing at their weakness and sharing the joke with the interviewer:

I was told it wasn't going to hurt, so I thought I could handle that. [laughter] ... As long as there's no pain involved, I can handle it. (UKPV5)

because I have made a fuss about the mammograms ... the first thing [the consultant] said to me was, he said, um, he said, I know how much you love the mammogram [laughter] . . (UKPV22)

A radiographer described the use of humour as a coping strategy, referring to a well-worn joke on preparatory exercises. $^{2}$

A way, I think, women deal with it is they - we make it comical. You know ... comedians talking about, oh, my mammogram, slamming your ... breast in a fridge door. (R2)

Other patients took on the role of the stoic - the strong woman, able to endure:

[I have] quite a deep sense of taking pain, it doesn't, you know, I never even have an injection to have my teeth filled. ...

The qualification of this, however, suggests endurance does not come easy:

because you've got to get on with it, you might as well steel yourself, and it's only for a second isn't it? (UKHV8)

Others were more downbeat about their capacity to endure:
I can't say the experience was very, ah ... encouraging you to really keep up with it, you know. ... You weigh your choices, you know, you want to take care of yourself, so you just do it, but it's a matter that you must do it, even though you suffer. (UKPV29)

they always have to press ... compress it so hard and ... hold your breath and just pray that they hurry up and take that picture. [laughs] (USPV278)

Such acceptance of pain - as a duty, to oneself and to the nation (Howson 1999; Griffiths et al. 2010) - appears to help preserve dignity and a measure of control. Even the woman who used the most extreme language about pain also embraced the story of endurance and understatement:

I find mammograms absolutely, incredibly painful ... oh, the agony! ... I have it done, because I think it's important to have it done, but I don't enjoy it. (UKHV6)

The persona of the negotiator appears rarely (although ACS, e.g., does recommend talking to the technician if feeling pain). One US participant recounted her successful challenge - more in the style of a battle than a negotiation:

... I look at the lady and I said, you, if you want to save your million dollar machine, you need to not press it down anymore ... She said, OK, well, I'll, you know, I'll do the, tell the doctor. You tell doctor to come on over here ... he's got stuff that can be mashed too, you know, it's not fair. So. (USHV296)

But for the most part, patients seem to reserve their critical comments for outside the clinic - and for the system in general - from sweeping generalisations like 'Everybody hates the mammogram' (UKPV6) to a plea for women to have 'the right to have a procedure that's not always uncomfortable for them' (USPV278) - rather than the individual encounter. The particular sub-theme of whether the mammogram would be tolerated if men were subjected to it is noted in the literature (Poulos \& Llewellyn 2005) and is intermittently developed on various blog and chat sites ${ }^{3}$ but not as any kind of organised protest.

While patients seem to show little appetite to negotiate a better deal, either on the general or the individual scale, there is however an important role for interpersonal negotiation in the clinical encounter as discussed below.

${ }^{3}$ For example, http://www.humorcolumnist.com/mammogram.htm; http://www.netfunny.com/rhf/jokes/02/Jun/mammogram.html

${ }^{2}$ See, for example, http://www.unexplained-mysteries.com/forum/index .php? showtopic $=9571$ 


\section{The professionals' positioning}

Studies carried out in a variety of locations have noted variations in patents' perceptions of staff behaviour as crucial to their experience of pain or discomfort (Aro et al. 1996; Bruyninckx et al. 1999; Engelman et al. 2006; Wiratkapun et al. 2006; Davey 2007; Almog et al. 2008). One such (Van Goethem et al. 2003) not only sought the opinions of patients on staff, but also the views of radiographers on patients, and found poor correlation between the radiographers' views about the patients' experience and patients' own accounts.

Patients' accounts indicate variable radiographer/ patient relationships, often shaped not so much by verbal communication as by the enacted physical relationship body language and sensitivity about level of compression. Thus, a patient observes of the screening service:

Some nurses are like 'oh, just squash it down' [laughs]

A bit gung-ho shall I say. (UKHV2)

while a cancer patient commented on how her second diagnostic mammogram was more comfortable than the first:

She did it very gradually, a bit more considerate .. . she could sense that my threshold had been reached. (UKPV2)

A radiographer acknowledging the variation explained it as an unfortunate hangover from an older tradition:

it does all come down to training. There are pockets of people who still firmly believe that you should give as much pressure as you possibly can. . . I wouldn't say that with the modern equipment it gets you a better image. And all it's doing is giving that woman a worse experience. (R2)

Her own practice appeared to recognise the need for a physically-mediated rapport with the patient:

When you're training someone, you tell them what the minimum is, but then they have to very much get used to the feel of the breast and the look of the woman. So you're feeling the tautness of the breast, but looking at the woman's face.

Official guidance to radiographers (Breast Screening Programme 2002) stresses the importance of patientradiographer interaction and provision of good verbal information prior to the procedure, but how or if this is to be structured into routine procedures is unclear. Thus, current policies put a great weight of responsibility on individual professional staff. Radiographers need skills in physically guiding and talking anxious patients through the procedure, often under pressure of time:

you say, well, you know, we'll do it together. Let's just talk about it. You've only got six minutes, so you're manoeuvring them while you're saying that ... if you talk about it and tell them that, I control the footswitch, so at any time. ... I can stop it. I think that's the crucial thing. (R2)

In the diagnostic mammogram, there is more time for talk, while giving the same message.

This radiographer's offer to share power demonstrates a practical 'work-around' or adaptation of the findings of Kornguth et al. (1996) about how more control of the compression process could improve a patient's pain experience - a finding accepted in principle by Miller et al. (2008) in their Cochrane review but not deemed currently implementable.

Radiographers are in the invidious position of having to reconcile time limits (about 6 min per examination for screening services) and strictly monitored standards for a technically satisfactory image (in the UK NHS there are penalties for a technical recall rate exceeding $3 \%$ ) with vaguer recommendations to give more information and take a gentle and supportive approach. Performance moreover is judged on image quality not patient experience. Thus, for professionals as well as patients the mammogram makes considerable but little remarked personal/ professional demands.

\section{Absentees \\ Despite operators' persuasive techniques and fine judge- ments, the physical limits to amelioration remain: \\ ... when that's put in the vice, and that's wound down, it hurts, and I say to the girl, it hurts, she says, well it will do. And I've said to [the consultant], that hurts when they do that. He says, well it will. (UKPV34)}

Given such dour acknowledgement by the professionals, it seems not surprising that some women simply reject mammography, although their rationales extend beyond pain (Pfeffer 2004). Acceptance rates for UK screening programmes, for example, were about $73 \%$ in $2010-1011$, which leaves a sizeable minority who are not complying. Information on non-attenders is sparse, but pain and 'lack of respect' are commonly cited (Marshall 1994; Elwood et al. 1998; Johansson \& Berterõ 2003; Pfeffer 2004; Engelman et al. 2006; Barter-Godfrey \& Taket 2007). For those women who remain compliant, it takes a combined 
effort (of varying intensity) between themselves and professional staff to maintain the effectiveness of the technology.

\section{DISCUSSION}

These observations and findings suggest that for all its solid medical, public and technical credentials, mammogram technology depends substantially for its effectiveness on a number of social and cultural conventions, beliefs and contingently established working practices. These include the target populations' beliefs about responsible citizenship, their fear of disease, heightened awareness of risk and respect for medical authority. While this may apply to any widely used diagnostic technology, the tensions in enacting the procedure are particularly visible in mammography, given the lack of effective premedication and the high visibility and direct effect of the machine-driven compressive process. The literature suggests that pain acts as a disincentive in varying degrees to compliance with screening regimes and an additional anxiety for patients with suspected or known breast disease. This has implications for policy - particularly policies regarding: (1) sustaining a culture that enables (and ennobles) compliance; (2) reviewing arrangements for pain management; and (3) reassessing the place of alternative technologies in longer-term planning.

Given the authoritative 'truth discourse' offered in patient information leaflets and some older academic articles advising that pain is not a problem (Rutter et al. 1992), one aim of this paper has been to demonstrate that pain in mammography is an issue to be taken seriously. The academic literature cited provides evidence of concern in the medical profession as well as continuing divisions of opinion on 'what counts' as pain. Additionally, the qualitative data adduced here, although it cannot resolve disagreements about the prevalence of pain, does indicate the physical and mental stresses a mammogram can induce, and the personal and professional resources consumed in accomplishing satisfactory results. The paper has focused on two voices that may hitherto have been insufficiently heard in the context of this routine diagnostic examination - those of the patients (including screening patients) and the front-line professionals administering the procedure. Their comments suggest that concerns about pain may have been underestimated and deserve more systematic attention in the future.

Arguments for change based primarily on compassion and micro-level inefficiencies may however carry little weight against powerful arguments for maintaining the status quo. The mammogram meets technical criteria for an effective diagnostic tool and is established as part of the institutional framework of the public health services. There is technological lock-in to the extent of massive investment in equipment, staff training, improvements (such as digitisation) and development of professional expertise. Even were it less effective than it is, it would be hard to dislodge for these reasons. Equally important is the weight of medical authority promoting the mammogram in seamless alliance with cancer charities and patient groups, together with the special status of cancer as a 'dread disease' (Sontag 2006) adding to pressures for compliance. Patient organisations play an important role in promoting cancer awareness as well as in reinforcing medical authority. Their campaigns add to the macrolevel encouragement to populations to construct themselves as at risk of disease and take responsibility for managing their health (Rose 2001; Willis 2004, 2008; Kampf 2010) and graphically reinforce the messages of the importance of early detection and individual responsibility (Foskett 2000; Klawiter 2008). Gigerenzer (2010) has argued that the patient information available to women from all sources has led to their seriously overestimating the risk of cancer and the benefits of screening mammograms (Schwartz et al. 2004; Jepson et al. 2007). A side effect of the generally welcome high level of activism and awareness of cancer may thus be an imbalance of information, tending to boost public acceptability of the mammogram and downplay its shortcomings.

These influences may account for the extent to which the pain issue is pushed to the margins. While in other areas of medicine pain mitigation has been a major objective (Nettleton 1989; Taddio et al. 1995, 2009; Ipp 2004), there is some (largely anecdotal) evidence of undervaluing pain in relation to diagnostic methods for other forms of cancer (Singleton \& Michael 1993); see also the minority view in the patient database on prostate biopsy on the website Healthtalkonline. ${ }^{4}$ Health service providers judge acceptability of the mammogram on compliance rates and (at the local level) formal patient complaints. Despite the negative evidence on patient experience, very few women refuse a diagnostic mammogram and acceptance rates for UK screening programmes are sufficient to satisfy service providers (Breast Screening Programme 2006). Furthermore, the qualitative findings cited here indicate little appetite for radical action among patients, including those who express deep aversion to the process. And the substantial minority who simply stay away do not constitute a large enough number to trigger review of policy.

${ }^{4}$ See http://www.healthtalkonline.org/Cancer/Prostate_Cancer/Topic/ $1890 /$ 
There seems little prospect of early rescue via alternative imaging technologies (although women taking part in the tests of the new optics-based technology seemed to invest a good deal of hope in this). Karellas and Vedantham (2008) comment on the 'unfortunate' fact that 'the scientific community has been unable to overcome the vigorous breast compression needs of mammography' (p. 4881), but still envisage the mammogram (in its digital version) maintaining its leading position over the next 10 years, although increasingly being used in combination with other modalities. Developers of alternative breast-imaging modalities (such as optical imaging requiring little or no compression) concur with this multi-modality model, with the mammogram still in the lead, rather than replacement (Leff et al. 2008; Pogue et al. 2010). This cautious approach does include a space for research on further developing the whole raft of technologies, but offers mammogram-averse patients only faint comfort for the immediate future.

\section{CONCLUSIONS}

The muted level of dissent leads to the conclusion that the majority of women feel compelled (by fear or duty) to comply with the demands of mammogram technology, and with the help of their personal strategies and 'workarounds' developed with radiographers are complicit in sustaining the present unloved regime. The compulsion to self-surveillance, as responsible citizens and family members, combines with ready acceptance of the discourse on the risk of cancer and the benefits of mammograms to command their attendance as recommended at clinic or screening centre, working on themselves by further practices of the self as necessary. Exceptions to this are of course the important minority of those opting out of screening programmes altogether. It appears however that their numbers are insufficient to stimulate reforms. As long as the majority of patients and potential patients comply with the diagnostic regime proposed to them, their pain or discomfort will remain personal troubles not public issues (Mills 1959) and radical change cannot be expected.

This need not however rule out a greater commitment to mitigatory action, and I conclude with some possibilities for practical change suggested by this study.

1 Listening to the patient/consumer voice indicates that pain is an issue for many women undergoing mammograms, even though it may not affect their compliance. Recognising this in the tone and content of patient information and advising on ways to deal with it would show greater respect for patients than blanket reassurance, which too often appears in patient information. In the case of the NHS, this change would accord with their declared policy on providing balanced information rather than promotional literature (Breast Screening Programme 2006). Patient advocacy groups might also wish to consider whether a franker focus on pain could enhance their special role in providing support and lobbying for attention to patients' needs.

2 While there is general acceptance of the importance of the radiographer/patient relationship in producing good images with less patient discomfort, there appears to be scope for this to be better integrated into training programmes, formal working protocols, procedural guidelines and assessment criteria for radiographers' performance. Provision for skills and structures relevant to patient experience might usefully be included in all of these, in line with accepted policies for a partnership approach to patient/professional relations and recommendations already contained in staff guidance on the development of sympathetic relationships.

3 At the technical level, service providers and other funding bodies might wish actively to support the translation into practice of experimentally tested means of pain mitigation (including physical interventions, e.g., reduced compression), and ensure that implications for patient experience are routinely taken into account in considering need for upgrading equipment. Consideration of patient comfort might also be added to other well-acknowledged reasons (concerning radiation risks and technical limitations) for further developing and integrating into practice alternative or complementary imaging modalities.

Despite relatively high compliance levels, the mammogram is generally disliked and often feared, and evokes tensions between aversion to pain and commitment to responsible citizenship. While pain is only one of many issues involved in the complex interplay of ideologies, authorities, emotions and workplace negotiations that sustains mammography, its behavioural and emotional reach is broad. Greater and more structured attention to pain management has potential not only to improve the experience of patients and front-line professionals, but also to contribute to diagnostic effectiveness.

\section{ACKNOWLEDGEMENTS}

The author warmly acknowledges the help and support given by Dr Megan Clinch and likewise the cooperation, interest and helpful insights from scientific collaborators, patient advocates and research participants alike. 


\section{REFERENCES}

Almog R., Hagoel L., Tamir A., Barnett O. \& Rennert G. (2008) Quality control in a National Program for the Early Detection of Breast Cancer - women's satisfaction with the mammography process. Women's Health Issues 18, 110117.

American Cancer Society (ACS) (2014) How is a mammogram done? American Cancer Society.

Andrews F.J. (2001) Pain during mammography: implications for breast screening programmes. Australasian Radiology 45, 113-117.

Armstrong D. (1995) The rise of surveillance medicine. Sociology of Health and Illness 17, 393-404.

Armstrong K., Moye E., Williams S., Berlin J.A. \& Reynolds E.E. (2007) Screening mammography in women 40 to 49 years of age: a systematic review for the American College of Physicians. Annals of Internal Medicine 146, 516-526.

Aro A.R., Absetz-Ylöstalo P., Eerola T., Pamilo M. \& Lönnqvist J. (1996) Pain and discomfort during mammography. European Journal of Cancer 32, 1674-1679.

Barter-Godfrey S. \& Taket A. (2007) Understanding women's breast screening behaviour: a study carried out in South East London, with women aged 50-64 years. Health Education Journal 66, 335346

Baszanger I. (1998) Pain: its experience and treatments. Social Science and Medicine 29, 425-434.

Breast Screening Programme (2002) Information and Advice for Health Professionals in Breast Screening. NHSBSP Publication No 53. NHS Cancer Screening Programmes, Sheffield, UK.

Breast Screening Programme (2006) Screening for Breast Cancer in England: Past and Future. Publication No 61. NHS Breast Screening Services, Sheffield, UK.

BreastScreen Australia (2014) Step by step guide to mammography. Internet. Available at: http://www.cancerscreening.gov .au/internet/screening/publishing.nsf/ Content/how

Brett J., Bankhead C., Henderson B., Watson E. \& Austoker J. (2005) The psychological impact of mammographic screening. A systematic review. Psycho-Oncology 14, 917-938.

Brown P.R., Alaszewski A., Swift T. \& Nordin A. (2011) Actions speak louder than words: the embodiment of trust by healthcare professionals in gynaeoncology. Sociology of Health and Illness 33, 280-295.

Bruyninckx E., Mortelmans D., Van Goethem M. \& Van Hove E. (1999) Risk factors of pain in mammographic screening. Social Science and Medicine 49, 933-941.

Cartwright L. (1995) Screening the Body: Tracing Medicine's Visual Culture. University of Minnesota Press, Minneapolis, MN, USA.

Crowley-Matoka M., Saha S., Dobscha S.K. \& Burgess D.J. (2009) Problems of quality and equity in pain management: exploring the role of biomedical culture. Pain Medicine 10, 1312-1324.

Davey B. (2007) Pain during mammography: possible risk factors and ways to alleviate pain. Radiography 13, 229-234. Department of Health (2009) Be Active, Be Healthy: A Plan for Getting the Nation Moving. Department of Health, London, UK.

Department of Health (2010) Healthy Lives, Healthy People: Our Strategy for Public Health in England (CM7985). Stationery Office, Norwich, UK.

Doyle C.A. \& Stanton M.T. (2002) Significant factors in patient satisfaction ratings of screening mammography. Radiography 8, 159-172.

Elwood M., McNoe B., Smith T., Bandaranayake M. \& Doyle T.C.A. (1998) Once is enough - why some women do not continue to participate in a breast cancer screening programme. New Zealand Medical Journal 111, 180-183.

Engelman K.K., Cizik A.M. \& Ellerbeck E.F. (2006) Women's satisfaction with their mammography experience: results of a qualitative study. Women and Health 42, 17-35.

Foskett J. (2000) Problematizing biomedicine: women's construction of breast cancer knowledge. In: Ideologies of Breast Cancer: Feminist Perspectives (ed. Potts L.), pp. 15-36. MacMillan, London, UK

Gigerenzer G. (2010) Women's perception of the benefit of breast cancer screening. Maturitas 67, 5-8.

Gill R. (2000) Discourse analysis. In: Qualitative Researching with Text, Image and Sound (eds Bauer M. \& Gaskell G.), pp. 172-190. Sage, London, UK.

Griffiths F., Bendelow G., Green E. \& Palmer J. (2010) Screening for breast cancer: medicalization, visualization and the embodied experience. Health 14, 653-668.

Howson A. (1999) Cervical screening, compliance and moral obligation. Sociology of Health and Illness 21, 401-425.

Institute of Medicine (IoM) (2011) Relieving Pain in America: A Blueprint for Transforming Prevention, Care, Education, and Research. National Academies Press, Washington DC, USA.

Ipp M. (2004) Effect of choice of measlesmumps-rubella vaccine on immediate vaccination pain in infants. Archives of
Pediatrics and Adolescent Medicine 158, 323-326.

Jepson R.G., Hewison J., Thompson A. \& Weller D. (2007) Patient perspectives on information and choice in cancer screening: a qualitative study in the UK. Social Science and Medicine 65, 890-899.

Johansson I. \& Berterõ C. (2003) Getting no respect: barriers to mammography for a group of Swedish women. Health Care for Women International 24, 8-17.

Jørgensen K.J. \& Gotzsche P.C. (2004) Presentation on websites of possible benefits and harms from screening for breast cancer: cross sectional study. BMJ (Clinical Research Ed.) 328, 148.

Jørgensen K.J., Keen J.D. \& Gotzsche P.C. (2011) Is mammographic screening justifiable considering its substantial overdiagnosis rate and minor effect on mortality. Radiology 260, 621-627.

Kampf A. (2010) 'The risk of age'? Early detection test, prostate cancer and practices of self. Journal of Aging Studies 24, 325-334.

Karellas A. \& Vedantham S. (2008) Breast cancer imaging: a perspective for the next decade. Medical Physics 35, 48784897.

Kashikar-Zuck S., Keefe F.J., Kornguth P., Beaupre P., Holzberg A. \& Delong D. (1997) Pain coping and the pain experience during mammography: a preliminary study. Pain 73, 165-172.

Klawiter M. (2008) The Biopolitics of Breast Cancer: Changing Cultures of Disease and Activism. University of Minnesota Press, Minneapolis, MN, USA.

Kornguth P.J., Keefe F.J. \& Conaway M.R. (1996) Pain during mammography: characteristics and relationship to demographic and medical variables. Pain 66, 187-194.

Leff D., Warren O., Enfield L., Gibson A., Athanasiou T., Patten D., Hebden J., Yang G. \& Darzi A. (2008) Diffuse optical imaging of the healthy and diseased breast: a systematic review. Breast Cancer Research and Treatment 108, 9-22.

Marshall G. (1994) A comparative-study of re-attenders and non-re-attenders for 2nd triennial National Breast ScreeningProgram appointments. Journal of Public Health Medicine 16, 79-86.

Miller D., Livingstone V. \& Herbison P. (2008) Interventions for relieving the pain and discomfort of screening mammography. Cochrane Database of Systematic Reviews (1), CD002942.

Mills C.W. (1940) Situated actions and vocabularies of motive. American Sociological Review 5, 904-913.

Mills C.W. (1959) The Sociological Imagination. Oxford University Press, New York, USA. 
National Health Service (NHS) (2011) NHS Breast Screening. Department of Health, London.

Nettleton S. (1989) Power and pain: the location of pain and fear in dentistry and the creation of a dental subject. Social Science and Medicine 29, 1183-1190.

Petersen A. \& Bunton R., eds (1997) Foucault, Health and Medicine. Routledge, London, UK.

Pfeffer N. (2004) 'If you think you've got a lump, they'll screen you.' Informed consent, health promotion, and breast cancer. Journal of Medical Ethics 30, 227-230.

Pogue B.W., Leblond F., Krishnaswamy V. \& Paulsen K.D. (2010) Radiologic and near-infrared/optical spectroscopic imaging: where is the synergy? AJR. American Journal of Roentgenology 195, 321-332.

Potter J. (1996) Representing Reality: Discuourse, Rhetorc and Social Construction. Sage, London, UK.

Poulos A. \& Llewellyn G. (2005) Mammography discomfort: a holistic perspective derived from women's experiences. Radiography 11, 17-25.

Resnik D. \& Rehm M. (2001) The undertreatment of pain: scientific, clinical, cultural, and philosophical factors. Medicine, Health Care, and Philosophy 4, 277-288.

Rose N. (2001) The politics of life itself. Theory, Culture and Society 18, 1-30.

Rose N. \& Novas C. (2007) Biological citizenship. In: Global Assemblages (eds Ong A. \& Collier S.), pp. 439-463. Blackwell Publishing Ltd, Oxford, UK.
Roy S.C. (2008) Taking charge of your health: discourses of responsibility in English-Canadian women's magazines. Sociology of Health and Illness 30, 463477.

Rutter D.R., Calnan M., Vaile M.S., Field S. \& Wade K.A. (1992) Discomfort and pain during mammography: description, prediction, and prevention. BMJ: British Medical Journal 305, 443-445.

Scheper-Hughes N. \& Lock M.M. (1987) The mindful body a prolegomenon to future work in medical anthropology. Medical Anthropology Quarterly 1, 6-41.

Schwartz L.M., Woloshin S., Fowler F.J. \& Welch H.G. (2004) Enthusiasm for cancer screening in the United States. IAMA: The Journal of the American Medical Association 291, 71-78.

Screening for life.CA (2014) Breast cancer: mammograms. Available at http:// www.screeningforlife.ca/breastcancer screening/about-breast-cancer-screening/ mammograms\#does_hurt

Shilling C. \& Mellor P.A. (2010) Saved from pain or saved through pain? Modernity, instrumentalization and the religious use of pain as a body technique. European Journal of Social Theory 13, 521-537.

Singleton V. \& Michael M. (1993) Actor-networks and ambivalence general-practitioners in the UK Cervical Screening-Program. Social Studies of Science 23, 227-264.

Sontag S. (2006) Illness as Metaphor; and, AIDs and Its Metaphors. Picador, New York, USA.

Taddio A., Nulman I., Koren B.S., Stevens B. \& Koren G. (1995) A revised measure of acute pain in infants. Journal of Pain and Symptom Management 10, 456-463.

Taddio A., Chambers C.T., Halperin S.A., Ipp M., Lockett D., Rieder M.J. \& Shah V. (2009) Inadequate pain management during routine childhood immunizations: the nerve of it. Clinical Therapeutics 31, S152-S167.

Van Goethem M., Mortelmans D., Bruyninckx E., Verslegers I., Biltjes I., Van Hove E. \& De Schepper A. (2003) Influence of the radiographer on the pain felt during mammography. European Radiology 13, 2384-2389.

Werner A., Isaksen L.W.L. \& Malterud K. (2004) 'I am not the kind of woman who complains of everything': illness stories on self and shame in women with chronic pain. Social Science and Medicine 59, 1035-1045.

Willis K. (2004) Personal choice/social responsibility - women aged 40-49 years and mammography screening. Journal of Sociology 40, 121-136.

Willis K. (2008) 'I come because I am called': recruitment and participation in mammography screening in Uppsala, Sweden. Health Care for Women International 29, 135-150.

Wiratkapun C., Lertsithichai P., Wibulpolprasert B., Leelaswattanakul M., Detakarat J. \& Jungjai P. (2006) Breast pain and service satisfaction during digital mammography. Journal of the Medical Association of Thailand 89, 1864-1873. 